\title{
Streptococcal infection in a large general hospital
}

\author{
R. FREEMAN
}

From the Department of Bacteriology, University of Leeds

SYNOPSIS A report of a sustained increase in streptococcal sepsis in a large general hospital is presented. Attempts to define the cause are detailed, and the possible nature and consequences of it are discussed.

In the latter half of 1969 it was thought that an increasing number of beta-haemolytic streptococci were being isolated in this department, which deals with specimens from the Leeds General Infirmary. At the same time several cases of severe sepsis due to these organisms were treated. This study was prompted by these events.

\section{Methods and Investigations}

The first investigation was a retrospective survey to establish if the isolations of these organisms had in fact risen. Records were available for the last six months of 1969 , and these were compared with the records for the same period in 1963. (Records for the intervening years were not suitable.) The information collected was classified as follows.

\section{isOlations PER MONTH OF Streptococcus pyogenes}

Any beta-haemolytic streptococcus which was sensitive to the appropriate concentration of bacitracin was taken to be Streptococcus pyogenes, although in many cases the Lancefield group had been formally determined as well.

ISOLATIONS PER MONTH OF Staphylococcus aureus This term includes any coagulase-positive staphylococcus.

The sources of the isolated streptococci were noted.

The stated sensitivity of the isolated streptococci to penicillin and the tetracyclines was noted.

\section{Results}

The isolations per month of Streptococcus pyogenes are shown in Fig. 1 and for Staphylococcus aureus in Figure 2.

The sources of the isolated streptococci were Received for publication 10 September 1970.

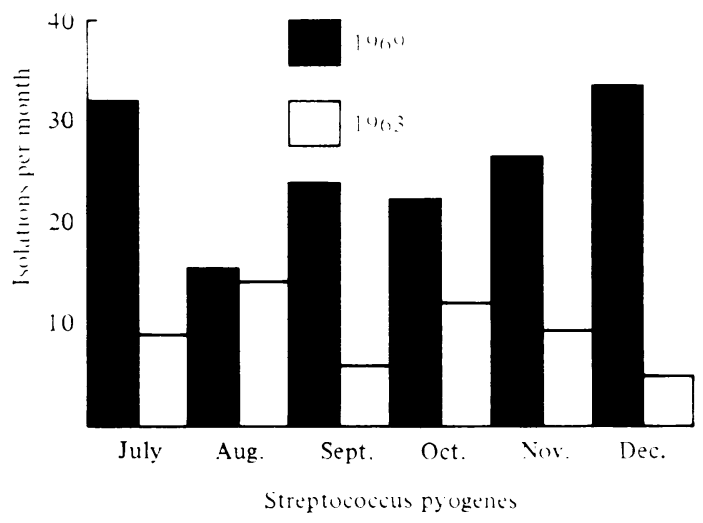

Fig. 1.

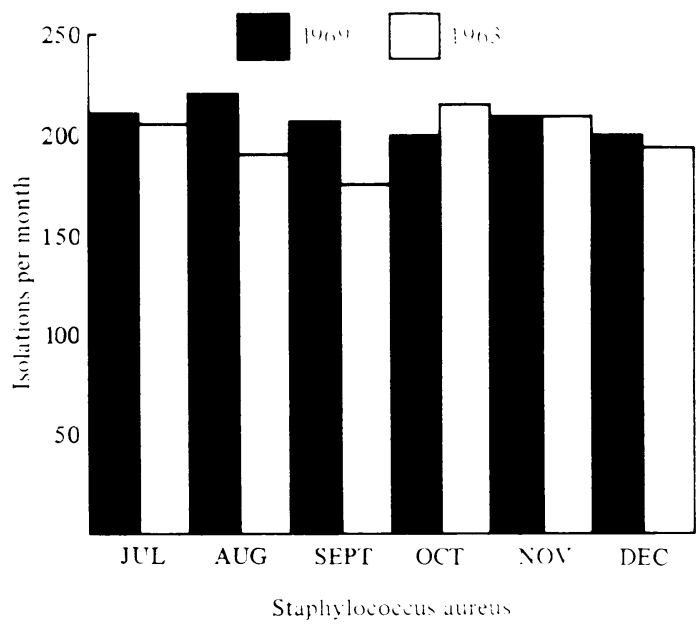

Fig. 2. 
divided into two groups: (1) streptococci from the upper respiratory tract and (2) from pathogenic sites. The latter included such sites as wounds, abscesses, etc, and any site where no innocent explanation could be given for the presence of the streptococcus. Seventeen cases from which streptococci were consecutively isolated were abscesses (6 cases), wounds following abscess (4 cases), pyoderma (6 cases), and myringotomy ( 1 case).

Figures 3 and 4 compare the two periods surveyed, dealing with isolations from the upper respiratory tract and the pathogenic sites in that order.

No streptococci were found which were resistant

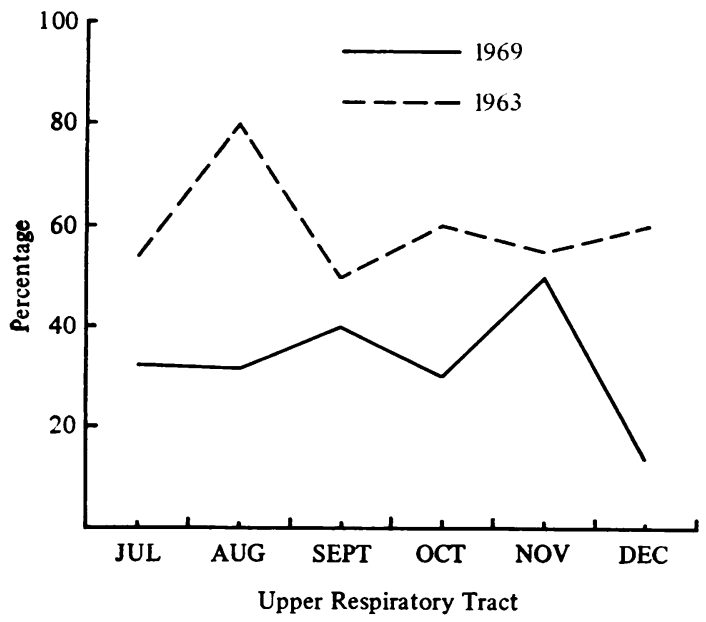

Fig. 3 .

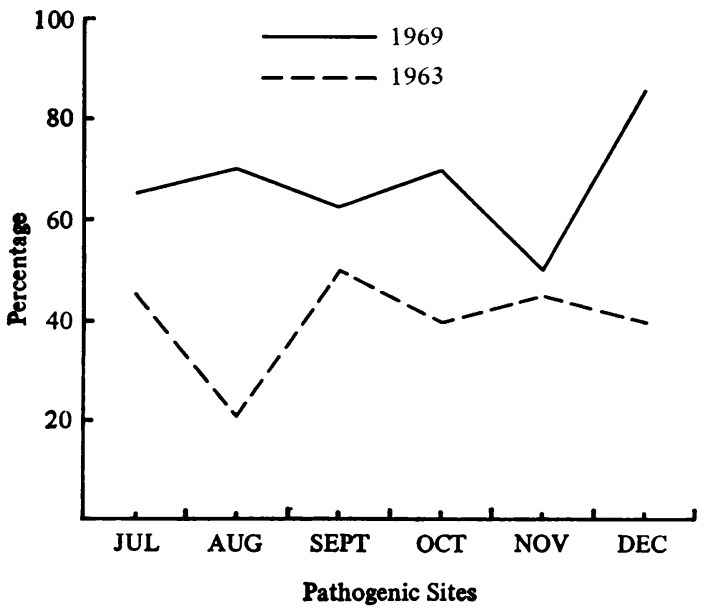

Fig. 4. to penicillin, and the resistance to tetracyclines in 1963 was $2 \%$ and in $19694 \%$. It can be seen from these results that the number of streptococci isolated per month from group 1 has indeed risen, whereas the number of staphylococci from similar sources has not risen to the same extent. Also notable is the finding that the source of the streptococcus is now more likely to be a non-respiratory one. There does not appear to have been any dramatic rise in the resistance of the streptococci to tetracyclines.

Although the rise in isolations had persisted for at least six months, and despite the initial evidence, it was decided to explore the two obvious possibilities, namely, an epidemic type of streptococcus and antibiotic resistance. At this time it became clear that almost all the cases were those seen in the Casualty Department. Accordingly, details of these patients were recorded over the two-month period 1 January 1970 to 28 February 1970 inclusive.

In all, 57 cases of sepsis involving a streptococcus of Lancefield group A were available for study in that period. The cases fell into two groups: (1) Streptococcus pyogenes the only organism isolated, (2) Streptococcus pyogenes and Staphylococcus aureus both isolated, each in heavy growth.

To decide the significance of the staphylococcus the swab from the lesion was inoculated onto the medium and three successive subinoculations were made from this site with a wire loop. The loop was flamed between streaks. Any subsequent growth of staphylococci was then assessed as follows: growth on first streak only + ; growth on first and second streaks ++ ; and growth on all three streaks +++ . Only specimens giving a +++ growth of staphylococci have been included in this survey as having a significant growth of these organisms.

For each of the two groups of cases the age and sex of the patient; sensitivity of the streptococcus to tetracyclines; and in cases which had yielded a staphylococcus, the sensitivity of this organism to penicillin, were noted. The results are set out in Tables I and II.

It must be noted that the 'mixed' cases, ie, ones in which both organisms were isolated, were not confined to superficial lesions. Heavy growths of 'mixed' type were obtained on several occasions from abscesses.

Two further investigations were carried out at this time: first, 18 consecutively isolated streptococci were typed using the precipitation and agglutination techniques (Table III). Finally, a survey was carried out in the Casualty Department over the same period to determine the age and sex distribution of pure staphylococcal sepsis, and the sensitivity of the causative organisms to penicillin. All sensitivities were estimated by disc-diffusion methods. 


\begin{tabular}{|c|c|c|c|c|c|c|}
\hline Total no. of cases & . & . & . & .. & $\cdots$ & 33 \\
\hline Age distribution & .. & .. & .. & .. & .. & . . Table IV \\
\hline Males . & .. & . & .. & .. & . & . 18 \\
\hline Females & . & .. & .. & .. & .. & $\ldots$ \\
\hline No. of strains resis & to $t$ & acyc & & .. & .. & .. \\
\hline
\end{tabular}

Table I Cases yielding a pure growth of Streptococcus pyogenes

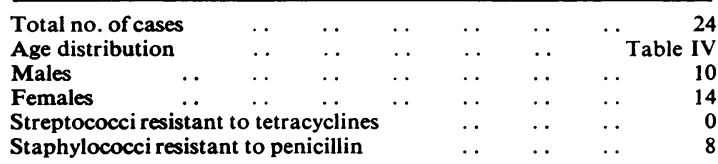

Table II Cases yielding mixed growth of Streptococcus pyogenes and Staphylococcus aureus

\begin{tabular}{lll}
\hline Strain No. & M Type & TType \\
\hline 1 & $-^{1}$ & $3 / 13 / 3264 \mathrm{~B}$ \\
2 & 12 & 12 \\
3 & - & $3 / 13 / 3264 \mathrm{~B}$ \\
4 & - & $5 / 27 / 44$ \\
5 & - & $3 / 13 / 3264 \mathrm{~B}$ \\
6 & - & $3 / 13 / 3264 \mathrm{~B}$ \\
7 & - & Imp. 19 \\
8 & - & - \\
9 & - & $3 / 13 / 3264 \mathrm{~B}$ \\
10 & - & $5 / 27 / 44$ \\
11 & - & $3 / 13 / 3264 \mathrm{~B}$ \\
$12^{2}$ & - & $3 / 13 / 3264 \mathrm{~B}$ \\
$13^{2}$ & - & 9 \\
$14^{2}$ & - & 28 \\
$15^{2}$ & 12 & 12 \\
$16^{2}$ & 17 & 12.19 \\
18 & & \\
\hline
\end{tabular}

Table III Serological types of 18 consecutively isolated streptococci

${ }^{1}$ Denotes that no reaction was obtained and that the streptococcus is untypable using present antisera.

'Denotes a streptococcus from a mixed type of infection.

\section{Discussion}

The results from this investigation show that streptococcal sepsis is increasing in the area covered by this hospital's Casualty Department. The streptococcal infections which are increasing seem to be of a non-respiratory type (Figs. 1-4), and an analysis has shown that two types of cases exist: (1) pure streptococcal sepsis, and (2) a mixed infection due to the streptococcus and the staphylococcus together. These cases may have differing age incidences which appear distinct from that of pure staphylococcal sepsis (Tables IV and V).

Analysis of the streptococcal types involved has been unhelpful. Although many of the typed streptococci were found to be of the same $T$ type (3/13/3264B) this is in fact the common type found in skin and soft tissue infections and is thus epidemiologically of little significance (Parker, other authors,

\begin{tabular}{|c|c|}
\hline Age Group (yr) & No. of Lesions \\
\hline \multicolumn{2}{|c|}{ Pure streptococcal sepsis } \\
\hline $0-1$ & 0 \\
\hline $1-5$ & 1 \\
\hline $5-10$ & 2 \\
\hline $10-20$ & 11 \\
\hline $20-30$ & 6 \\
\hline $30-40$ & 6 \\
\hline $40-50$ & 2 \\
\hline $50-60$ & 3 \\
\hline $60+$ & 2 \\
\hline Total & 33 \\
\hline \multicolumn{2}{|c|}{ Mixed streptococcal and staphylococcal sepsis } \\
\hline $0-1$ & 0 \\
\hline $1-5$ & 0 \\
\hline 5-10 & 7 \\
\hline $10-20$ & 1 \\
\hline $20-30$ & 4 \\
\hline $30-40$ & 2 \\
\hline $40-50$ & 3 \\
\hline $50-60$ & 1 \\
\hline $60+$ & 1 \\
\hline Total & 24 \\
\hline \multicolumn{2}{|c|}{ Pure staphylococcal sepsis } \\
\hline $0-1$ & 15 \\
\hline $1-5$ & 41 \\
\hline $5-10$ & 18 \\
\hline $10-20$ & 64 \\
\hline $20-30$ & 103 \\
\hline $30-40$ & 38 \\
\hline $40-50$ & 25 \\
\hline $50-60$ & 11 \\
\hline $60+$ & 8 \\
\hline Grand total & 396 \\
\hline
\end{tabular}

Table IV Age incidences of septic lesions

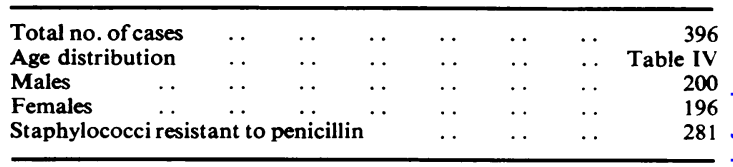

Table V Pure staphylococcal sepsis

1957). Similarly, although much reported in recent years, tetracycline resistance appears to have played little part in the rise (Robertson, 1965 and 1968).

However, two interesting new points do emerge. First, the finding of these two types of streptococcal infection, viz, pure and mixed, is not an unknown state of affairs and seems to have been common on many years ago (Lowden, 1955). It does appear to have become rarer in the more recent past and now, in this area at least, common again. The change from the universal use of penicillin to the more specifically antistaphylococcal drugs to combat resistant staphylococci is a possible explanation which would merit further investigation.

The second point is related to the first. The staphylococci present in the mixed type of infection show a high rate of sensitivity to penicillin (16 out of 24 are sensitive), whereas the staphylococci in pure staphylococcal sepsis are highly resistant to this 
drug (only about $30 \%$ are sensitive). There is possibly something unusual about these staphylococci, and it has recently been suggested that certain staphylococci, notably of phage type 71 , produce substances which are streptocidal (Dajani and Wannamaker, 1969). It remains a possibility that the converse may be true and more work is needed to elucidate this relationship.

Finally, a warning note should be added. Recent work in America has revealed disturbing aspects of streptococcal skin and soft tissue sepsis. The main facts seem to be that poststreptococcal diseases, eg, nephritis, are not uncommon sequelae, and that the conventional antibody test (the antistreptolysin $\mathbf{O}$ titre) may not reveal this (Wannamaker, 1970).

The situation reported in this article is not therefore merely a problem for the casualty department, but possibly a much more general and serious one (note in this context a recent Communicable Diseases Report, 1970).

It is a pleasure to thank Dr G. B. Ludlam and his staff at the Public Health Laboratory, Leeds, for the serological typing of Streptococcus pyogenes. My colleagues in the Department of Bacteriology, University of Leeds, deserve my thanks for advice and encouragement. Mr C. G. Burton gave valuable technical assistance.

\section{References}

Communicable Diseases Report (1970). Outbreaks of streptococcal skin sepsis, pp. 2-3. Central Public Health Laboratory, London.

Dajani, A. S., and Wannamaker, L. W. (1969). Demonstration of a bactericidal substance active against beta-haemolytic streptococci in supernatant fluids of staphylococcal cultures. J. Bact., 97, 985-991.

Lowden, T. G. (1955). Sepsis and antibiotics. In The Casualty Department, pp. 1-11. Livingstone, Edinburgh.

Robertson, M. H. (1965). Beta-haemolytic streptococci in South-west Essex, with particulat reference to tetracycline resistance. Brit. med. $J, 2,569-571$.

Robertson, M. H. (1968). Tetracycline-resistant streptococci in Southwest Essex: a continuing survey. Brit. med. J., 3, 349-350.

Williams, R. E. O. (1957). Serotypes of Streptococcus pyogenes: their relative prevalence in England and Wales, 1952-1956. Mth. Bull. Minist. Hlth Lab. Serv., 16, 163-172.

Wannamaker, L. W. (1970). Differences between streptococcal infections of the throat and of the skin. New Engl. J. Med., 282, 2331 and $78-85$. 